\title{
Chemokine responses in schistosomal antigen-elicited granuloma formation*
}

\author{
BO-CHIN CHIU ${ }^{1} \&$ STEPHEN W. CHENSUE ${ }^{1,2}$
}

${ }^{1}$ Department of Pathology, University of Michigan Medical School, Ann Arbor, MI and ${ }^{2}$ Department of Pathology and Laboratory Medicine, Veterans Affairs Healthcare System, Ann Arbor, MI, USA

\begin{abstract}
SUMMARY
Host immune systems have evolved specialized responses to multicellular parasites. This is well represented by the type 2 granulomatous response to Schistosoma mansoni egg antigens, which is an eosinophil-rich inflammatory response mediated by Th2-associated cytokines. Using Ag-bead models of pulmonary granuloma formation in mice, we defined characteristic chemokine (CK) profiles in the granulomatous lungs. Our findings point to a role for $C$-C chemokine receptor-2 (CCR2) and CCR3 agonists such as monocyte chemotactic proteins (MCPs) 1/CCL2, 3/CCL7 and 5/CCL12 as important participants that are subject to regulation by Th2 cytokines interleukin (IL)-4 and IL-13. CCR4 and CCR8 agonists are also likely contributors. Analysis of CK receptor knockout mice revealed that CCR2 ligands (e.g. MCP-1 and 5) promoted early phase granuloma macrophage accumulation, whereas anti-MCP-3 (CCL7) antibody treatment abrogated eosinophil recruitment. CCR8 knockout mice also demonstrated impaired eosinophil recruitment but this appeared to be related to impaired Th2 cell function. Transcript analysis of $\mathrm{CD}^{+} T$ cells generated during schistosome granuloma formation failed to show biased CCR8 expression but, having a more limited receptor repertoire, these cells were likely more dependent on CCR8 ligands. Together, these studies indicate an intricate involvement of chemokines in various stages and aspects of schistosomal egg Ag-elicited granuloma formation.
\end{abstract}

Keywords chemokines, Th1, Th2, chemokine receptors, granulomas

Correspondence: Dr Stephen W. Chensue, Pathology and Laboratory Medicine 113, AAVAHS, 2215 Fuller Road, Ann

Arbor, MI 48105, USA (e-mail: schensue@med.umich.edu).

*Paper presented at the British Society for Immunology Congress, 'Chemokines and Leucocyte Migration In Parasitic Disease', 4-7 December 2001, Harrogate, UK.

Accepted for publication: 19 April 2002

\section{INTRODUCTION}

A central pathological manifestation of schistosomiasis mansoni is the granulomatous response to tissue-deposited parasite ova. The efforts of a number of investigators over the last half-century have revealed much concerning the immunologic basis of this reaction. The schistosome egg granuloma is now known to be a hypersensitivity-type, T cell-mediated inflammatory reaction to ova-derived antigens $(1,2)$, which is modified by the duration of infection and immune status of the host (3). It is also recognized that cytokines associated with Th2 cells [interleukin (IL)-4, IL-5 and IL-13] contribute significantly to the development of these lesions (4-11). The Th2-mediated reaction is thought to be less destructive to bystander tissue than Th1 inflammation (12); however, the type 2 response is associated with florid fibrosis that seriously compromises organ function (13).

Presumably, the molecular signals involved in schistosome egg granuloma formation must include innate and adaptive components. While Ag-elicited $\mathrm{T}$ cell-derived cytokines provide important amplifying signals during granuloma formation, they probably are not the earliest initiators. Innate immune signals must strike the 'spark' that begins local cell mobilization, then adaptive immune cytokines help to sustain the lesion and dictate any sequelae (e.g. fibrosis). Early initiation factors are thought include the induction of adhesion and chemotactic molecules that localize the $\mathrm{T}$ cells required for subsequent waves of amplification. Subsequent T cell-mediated amplification would again probably involve induction of additional chemotactic cytokines that would further promote leucocyte infiltration. Thus, chemotactic factors presumably participate extensively in the granuloma, and the superfamily of structurally related chemotactic polypeptides, known as chemokines (CK), may be particularly important. More than 50 human $\mathrm{CK}$ and nearly as many murine homologueues have been described and reviewed (14-16). Chemokines exert their effect through guanosine nucleotide-protein coupled receptors, a superfamily of related receptors that are involved in transduction 
of a broad spectrum of extracellular stimuli, such as hormones, neurotransmitters, CKs, odourants and light. Analysis of CK receptor expression by leucocytes revealed that subtypes of receptors are expressed to different degrees by different cell types and thereby dictate responsiveness to particular CKs. For example, neutrophils strongly express CXCR1 and CXCR2 making them most responsive to ELR + CXC ligands, whereas eosinophils appear more responsive to CCR3 ligands. Another aspect of CK receptor physiology is that their expression is subject to cytokine-mediated regulation $(17,18)$, which permits fine-tuning of cellular responses based upon organ location and host immune status. There is circumstantial evidence for CK participation in granuloma formation but, due to the numbers and functional redundancy of CK and the complexity of models of granulomatous conditions, detailed analyses are challenging at best.

\section{ANTIGEN-BEAD MODELS OF GRANULOMA FORMATION}

Granuloma formation during active schistosome infection is an asynchronous affair making it difficult to study discrete stages of the response. Ova can be isolated and embolized to the lungs to establish a synchronized reaction, but events may vary depending on egg quality and reactions cannot be easily controlled, making comparison with other responses difficult. In order to provide a systematic approach to the study of granuloma formation, we developed standardized models of synchronous pulmonary granuloma formation using antigen-coated agarose beads $(19,20)$. In brief, after a 14-20 day sensitization period with Mycobacteria bovis purified protein derivative (PPD) or Schistosoma mansoni eggs, mice are challenged intravenously with beads coated with mycobacterial PPD or soluble schistosomal egg antigen (SEA), respectively. The beads embolize to the pulmonary microvasculature where they elicit multiple granulomatous foci. Compared to nonantigen coated control (CON) beads, the PPD and SEA beads induce florid granulomas with cellular compositions comparable to human lesions evoked during $M$. tuberculosis and $S$. mansoni infection (Figure 1). Histologically, all of the granuloma types contain macrophages, giant cells and lymphocytes, but the SEA granulomas are distinguished by a significant component of eosinophils and hence very closely resemble the reaction elicited by native schistosome eggs. The PPD- and SEA Ag-bead lesions show similar growth kinetics (Figure 2). There is an early rapid recruitment phase (1-2 days) with maximal size reached by day 4 . This is followed by a period of sustenance before finally entering a resolution phase at 8 days and beyond. A sequential analysis of cytokines produced by isolated granulomas reveal patterns of expression that are consistent with type 1 (Th1) and type 2 (Th2) associated (a)
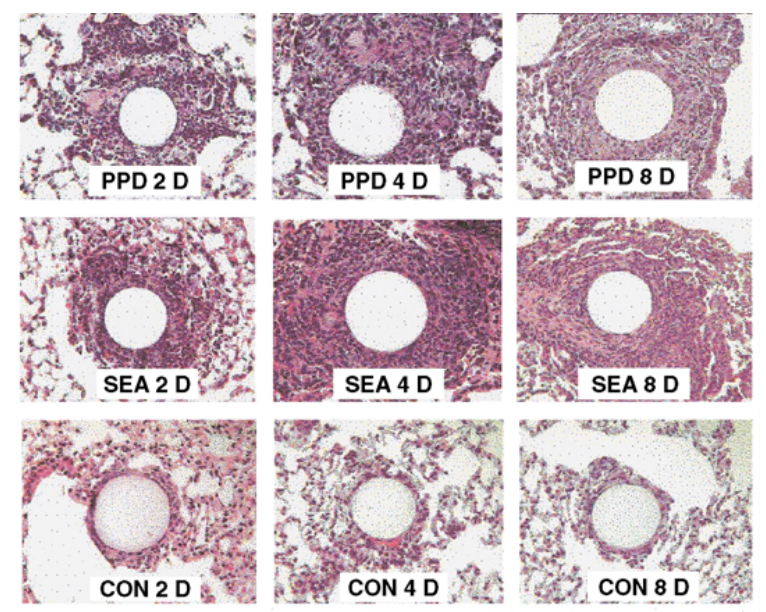

(b)

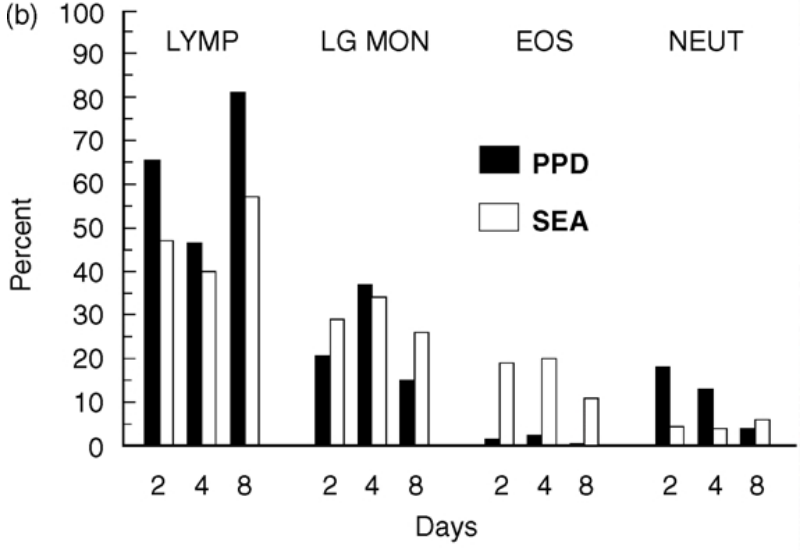

Figure 1 Histological appearance and cellular composition of experimental mycobacterial and schistosomal antigen-bead elicited pulmonary granulomas. (a) Type 1 mycobacterial purified protein derivative (PPD), type 2 schistosomal soluble egg antigen (SEA) and control $(\mathrm{CON})$ antigen-uncoated bead granulomas on days 2 , 4 and 8 of development. (b) Cellular differentials of dispersed PPD and SEA bead granulomas. LYMP, lymphocytes; LG MON, large mononuclear cells; EOS, eosinophils; NEUT, neutrophils.

cytokine profiles (Figure 3). Thus, Ag-coated bead models appear representative of the native granulomatous conditions and provide the means to monitor inflammatory events in a highly controlled fashion. Using this approach, we compared CK expression during type 2 SEA-elicited and type 1 mycobacterial PPD-elicited granuloma formation. Functional analyses were also performed using CK receptor knockout mice and in vivo anti-CK antibody treatments.

\section{CHEMOKINE AND CHEMOKINE RECEPTOR TRANSCRIPT PROFILES DURING AG-BEAD GRANULOMA FORMATION}

In order to correlate inflammatory events with CK expression, we surveyed transcript expression of $25 \mathrm{CKs}$ during 
Table 1 Relative expression of chemokine transcripts during type 1 mycobacterial and type 2 schistosomal Ag-bead granuloma formation

\begin{tabular}{llll}
\hline Ligands for: & Type 1 (PPD) & Type 2 (SEA) & Time of appearance \\
\hline CXCR2 & ++++ & ++ & Early \\
CXCR3 & ++++ & + & Early \\
CCR1 & ++++ & + & Early \\
CCR5 & ++++ & ++ & Early-mid \\
CCR2 & +++ & ++++ & Early-late \\
CCR3 & ++ & ++++ & Early-late \\
CCR4 & +++ & ++++ & Early-late \\
CCR6 & ++ & +++ & Late \\
CCR8 & ++ & ++++ & Mid-late \\
XCR 1 & +++ & ++ & Mid \\
CCR7 & - & - & NA \\
CXCR4 & - & - & NA \\
\hline
\end{tabular}

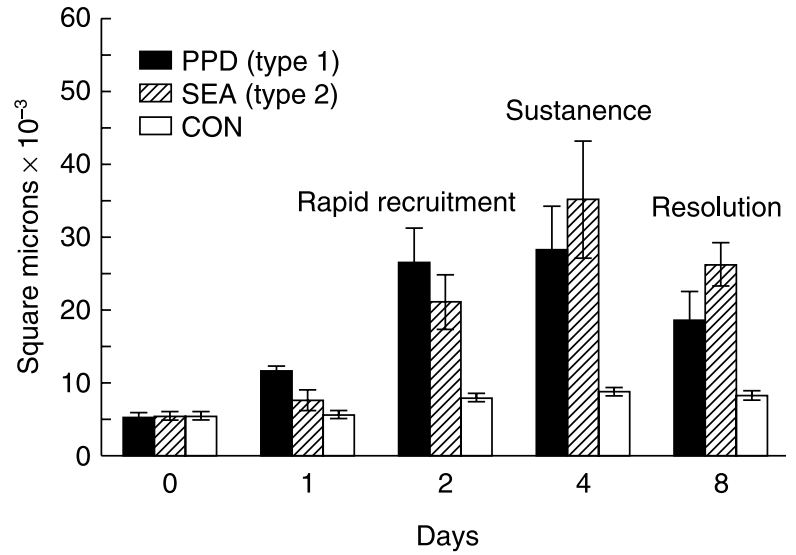

Figure 2 Stages and time course of mycobacterial and schistosomal antigen-bead elicited pulmonary granuloma formation. Synchronized type 1 PPD, type 2 SEA or control (CON) bead granulomas were elicited according to protocol and lesion areas were measured by computerized morphometry at the designated intervals.

the rapid recruitment, sustenance and resolution stages of the type 1 (PPD) and type 2 (SEA) responses using semiquantitative mRNA analysis of whole granulomatous lungs (21). In addition, we have similarly assessed expression of $10 \mathrm{CK}$ receptors. Characteristic type 1 and type $2 \mathrm{CK}$ profiles displaying both biased and shared CKs as well as distinct temporal expression patterns were observed (Table 1). These are discussed below. It should be noted that similar biased CK profiles have recently been reported by an independent laboratory using a schistosomal egg challenge model without and with IL-12 treatment to induce a polarized type 2 and type 1 responses (22).

As shown in Figure 4(a), CXCR2 and its ligands, MIP-2 and LIX were strongly expressed in the type 1 (PPD) response and associated with the early recruitment phase, but were more weakly expressed in the SEA response. This correlated well with the more dominant neutrophil influx
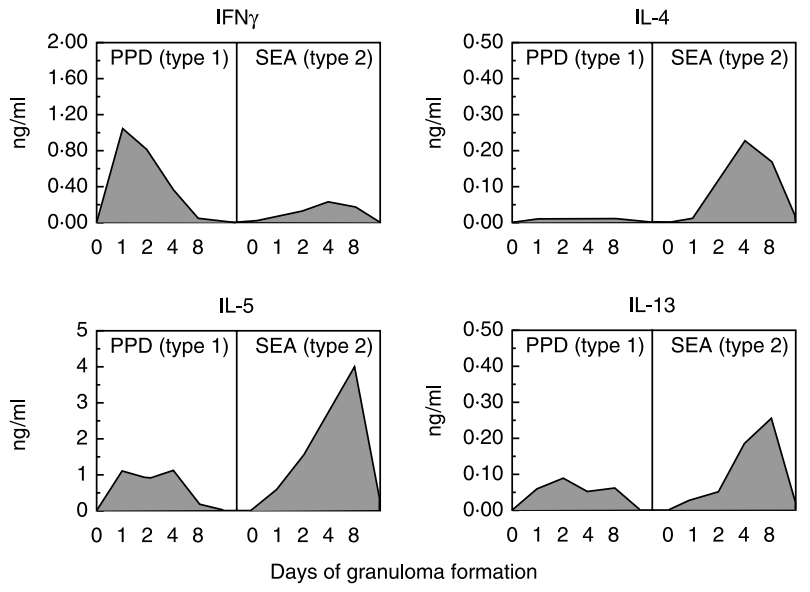

Figure 3 Time course and profiles of cytokine production by mycobacterial and schistosomal antigen-bead elicited pulmonary granulomas. Intact type 1 (PPD) or type 2 SEA bead granulomas were isolated from lungs at the designated intervals and cultured for $24 \mathrm{~h}$, then supernates were assayed for representative Th1 and Th2 related cytokines.

into PPD lesions at this time (Figure 1). CXCR3 ligands were similarly dominant in the early phase of the type 1 response but the receptor transcripts were expressed more strongly in the sustenance stages (Figure 4a). This receptor did not display type-specific restriction; however, CXCR3 may be expressed by a minor subpopulation of $\mathrm{T}$ cells and restricted expression could be obscured due to dilution among transcripts from other sites in the lung.

Transcripts for CCR1, CCR 5 and their ligands, MIP-1 $\alpha$, MIP-1 $\beta$ and RANTES, also displayed dominance in the type 1 response (Figure 4b). CCR1 is reportedly expressed by activated neutrophils, which may be partly responsible for the observed bias (18). Interestingly, the temporal expression of CCR 1 and CCR5 tended to parallel the expression of their respective ligands, MIP- $1 \alpha$ and MIP- $1 \beta$. These findings would predict that CCR1 ligands would 
(a)
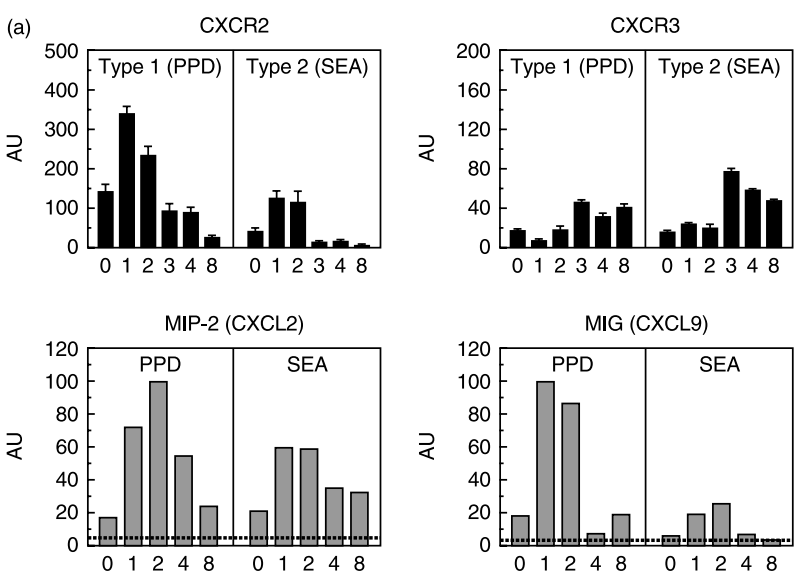

LIX (CXCL5)
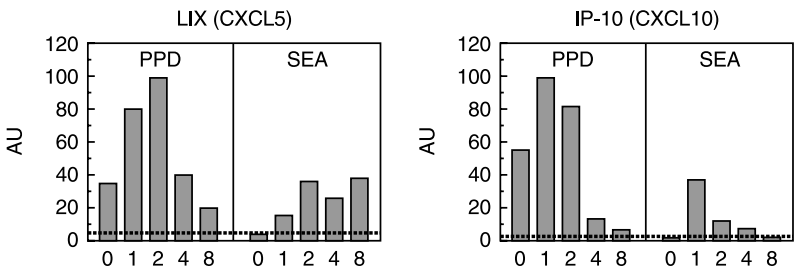

Days of granuloma formation
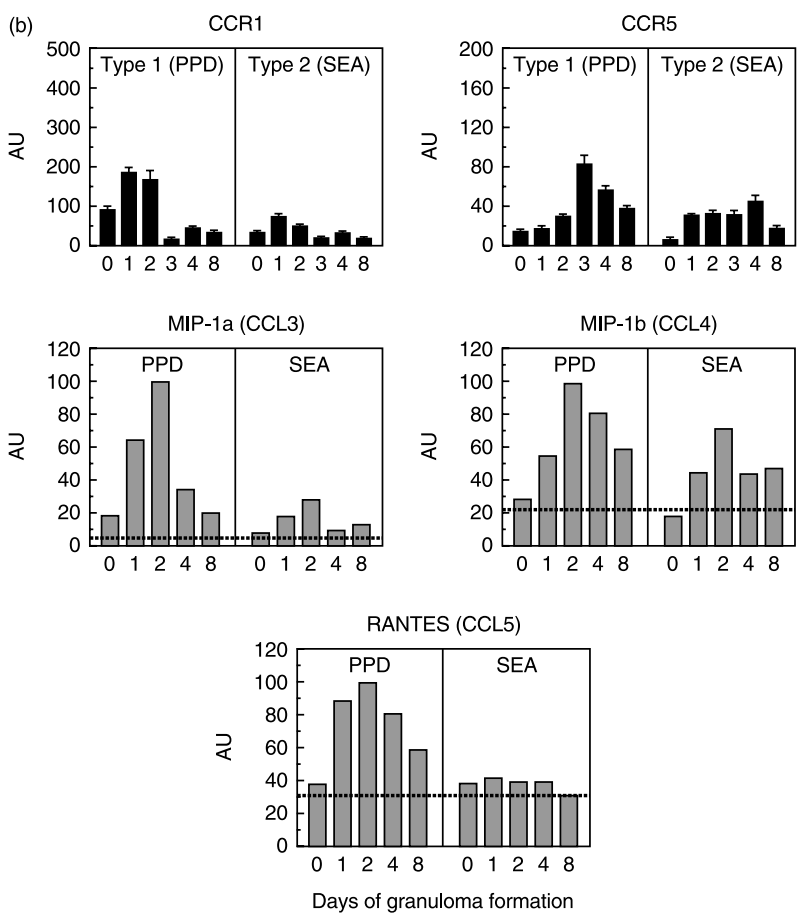

Figure 4 Time course of expression and relative levels of transcripts for chemokine receptors and representative chemokine ligands expressed in whole lungs during mycobacterial and schistosomal antigen-bead elicited pulmonary granuloma formation.

(a) CXCR2, CXCR3 and their ligands; (b) CCR1, CCR5 and their ligands. Corresponding ligand transcripts are shown immediately below the receptor. Midpositioned ligands are known to be highly promiscuous. Dashed lines are levels in naïve unchallenged lungs. Day 0 represents lungs of sensitized mice just prior to challenge.
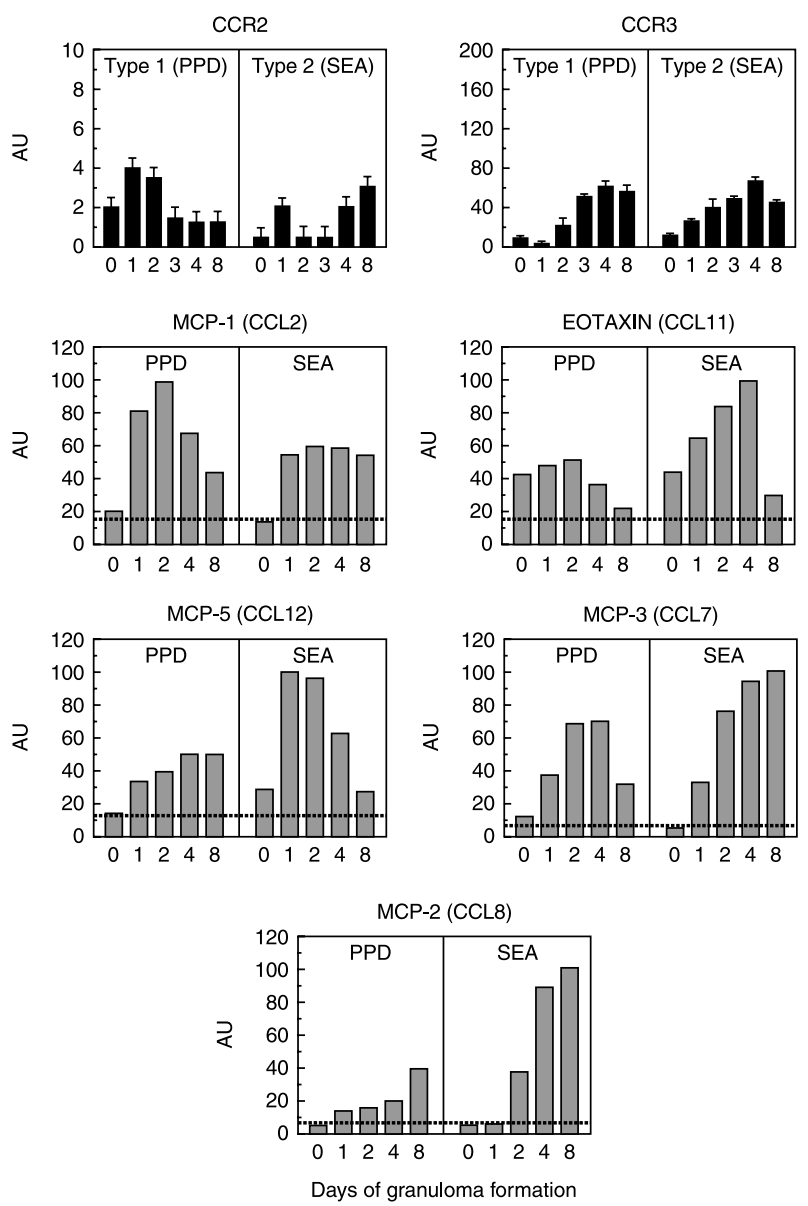

Figure 5 Time course of expression and relative levels of transcripts for chemokine receptors and representative chemokine ligands expressed in whole lungs during mycobacterial and schistosomal antigen-bead elicited pulmonary granuloma formation. Transcripts for CCR2, CCR3 and their ligands are shown. Corresponding ligand transcripts are shown immediately below the receptor. Midpositioned ligands are known to be highly promiscuous. Dashed lines are levels in naïve unchallenged lungs. Day 0 represents lungs of sensitized mice just prior to challenge.

participate to lesser degree in the SEA-elicited response and, indeed, we recently reported that mice with CCR1 knockout showed defects only in the type 1 PPD bead response (23).

As shown in Figure 5, CCR2 transcripts were modestly expressed with a partial type 1 biased expression. In addition, transcripts for the CCR2-specific ligand, MCP-1 (CCL2), were also detected in both responses, appearing as early as $1-2$ days with a slight type 1 predominance. The modest expression of CCR 2 may be related to downregulation as circulating monocytes move from blood to interstitial compartments (24). Interestingly, MCP-5 (CCL12) is highly homologous to MCP-1 (25) and its transcripts appeared in the early recruitment phase of the type 2 
response in a pattern similar to that seen for MCP-1 in the type 1 response. This suggests that MCP-5 provides an early monocyte recruitment function in the schistosomal response that MCP-1 provides in the mycobacterial response.

Transcripts for CCR3 were expressed mainly in mid to late stages of granuloma formation and did not display type specific bias (Figure 5). The latter was surprising, since CCR3 was expected to be dominant in the eosinophil-rich type 2 granuloma. Recent reports suggest that activated neutrophils and macrophages may express CCR3 $(18,24)$, but its functional participation in a Th1-mediated responses is still unclear. Overall, ligands with CCR3 or promiscuous CCR2/3 reactivity displayed biased expression in the type 2 (SEA) response. These appeared in mid to late stages of SEA-bead granuloma formation, which corresponds to the time of CCR3+ eosinophil accumulation and granuloma maintenance.

Since both CCR 4 and CCR 8 have been associated with Th2 cells (26-29), biased expression in the type 2 (SEA) response was initially predicted. However, CCR4 transcripts were expressed in both type 1 (PPD) and type 2 (SEA) responses in mid to late stages of granuloma formation with only a partial bias to the type 2 response. CCR 8 transcripts were also comparably expressed during both responses in the same period. This may indicate that these receptors are not polarized in vivo or that non-T cell populations obscured any underlying bias among Th cells. Activated NK and endothelial cells reportedly can express these receptors $(30,31)$.

As shown in Figure 6(a), transcripts for the respective ligands of CCR4 and CCR8 [i.e. MDC (CCL22) and TCA-3 (CCL1)] displayed a partial type 2 bias, but those for TARC (CCL17), which potentially binds to both receptors $(32,33)$, showed unbiased expression throughout early and late stages of inflammation. Interestingly, TARC contributes to the lymphoid response to Propionibacterium acnes granulomas and may therefore represent a granulomagenic CK (34).

As shown in Figure 6(b), CCR6 transcripts appeared in the mid to late stages of granuloma formation and displayed dominant expression in the type 1 response. This receptor has been reported on dendritic cells, B cells, memory T cells and neutrophils (35-38), all of which may take part in the inflammatory response. While the source of the biased expression is yet to be determined, the finding further underscores the different quality of schistosomal and mycobacterial lesions. Curiously, mRNA for LARC (CCL20), the only reported agonist for CCR6, was expressed in the late stage of granuloma formation with a partial type 2 bias, suggesting that this $\mathrm{CK}$ may not participate in cellular recruitment or rather contributes to the resolution stage of the lesion. (a)
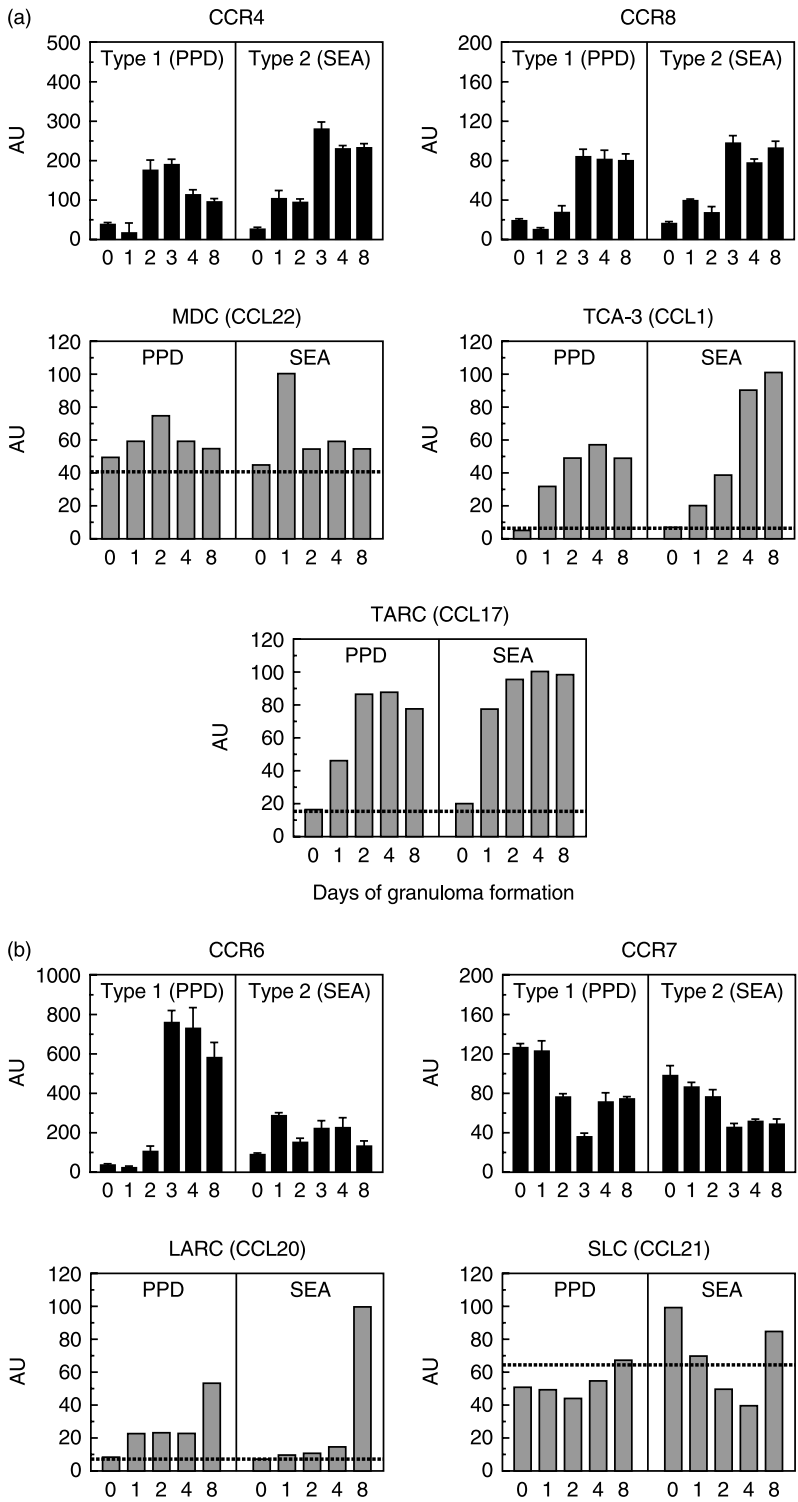

Days of granuloma formation

Figure 6 Time course of expression and relative levels of transcripts for chemokine receptors and representative chemokine ligands expressed in whole lungs during mycobacterial and schistosomal antigen-bead elicited pulmonary granuloma formation. (a) CCR4, CCR8 and their ligands; (b) CCR6, CCR7 and their ligands. Corresponding ligand transcripts are shown immediately below the receptor. Midpositioned ligands are known to be highly promiscuous. Dashed lines are levels in naïve unchallenged lungs. Day 0 represents lungs of sensitized mice just prior to challenge.

In our CK survey, a number of CKs were found to be constitutively expressed without induction during granuloma elicitation. These included stromal cell-derived factor$1 \alpha$ (CXCL12), lungkine (CXCL15), EB11 ligand chemokine (CCL19), secondary lymphoid tissue chemokine (SLC) 


\begin{tabular}{lll}
\multicolumn{1}{c}{ Early (1-2 d) } & \multicolumn{1}{c}{ Mid (2-4 d) } & \multicolumn{1}{c}{ Late (4-8 d) } \\
\hline \multicolumn{1}{c}{} & \multicolumn{1}{c}{} & \\
MCP-1(CCR2) & MCP-2 (CCR2/3) & MCP-2 (CCR2/3) \\
MCP-5(CCR2) & MCP-3 (CCR3) & MCP-3 (CCR3) \\
MDC (CCR4) & EOTAXIN (CCR3) & TCA-3 (CCR8) \\
TARC & TCA-3 (CCR8) & TARC (CCR4/8?) \\
(CCR4/8?) & TARC (CCR4/8?) & LARC (CCR6) \\
\hline
\end{tabular}

Figure 7 Major chemokines identified during critical stages of schistosomal egg antigen-elicited granuloma formation. Note that $\mathrm{CC}$ chemokines, monocyte chemotactic proteins (MCPs) and thymus activation related chemokine (TARC) are found at all stages.

(CCL21) and fractalkine (CX3CL1). Interestingly, many of these are thought to participate in homeostatic lymphoid circulation. As shown in Figure 6(b), transcripts for the ligand, SLC, showed no induction or declined, whereas the level of transcripts for its receptor CCR7 tended to decline with granuloma development probably reflecting dilution in the pool of mRNA derived from newly recruited cells. This finding supports the current notion that selected CKs may have designated inflammatory or noninflammatory functions $(39,40)$.

Figure 7 summarizes the potential CKs contributing to type 2 schistosomal egg Ag-elicited granuloma formation. It is noteworthy that most of these are beta or CC-type CKs that interact with receptors, CCR2, 3, 4 or 8 . In previous reports, we provided evidence that type 2 cytokines, IL-4 and IL-13, appear to amplify the production MCP-1, MCP3 and MCP-5, all CKs associated with this response $(41,42)$.

\section{FUNCTIONAL CONTRIBUTION OF CHEMOKINES TO SCHISTOSOMAL EGG ANTIGEN-ELICITED GRANULOMA FORMATION}

The above CK survey provided strong circumstantial evidence for $\mathrm{CK}$ participation in the type 2 schistosomal Ag-elicited granuloma, but we also performed a number of studies to test function using CK receptor knockout mice and in vivo antibody-mediated $\mathrm{CK}$ depletion. For example, mice with CCR2 knockout displayed a selective 50\% abrogation of macrophage accumulation primarily during the early recruitment phase of granuloma formation that was associated with corresponding reduction in lesion size (43) (Figure 8). This effect correlated precisely with the time of expression of the CCR2 ligands, MCP-1 and MCP-5, noted above. Thus, CCR2 ligands appeared to promote macrophage accumulation at least during the early recruitment
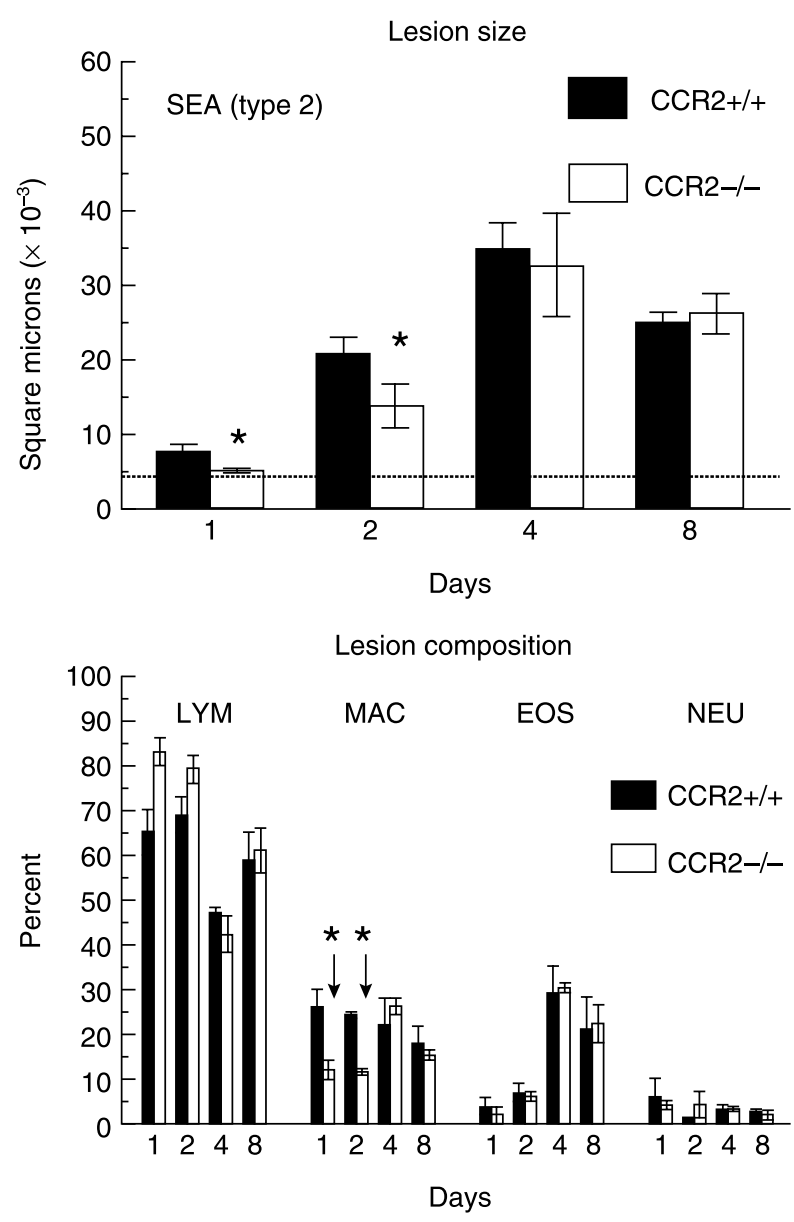

Figure 8 Effect of $\mathrm{CC}$ chemokine receptor 2 (CCR2) knockout on schistosomal egg antigen-elicited granuloma formation. Type 2 (SEA) bead granulomas were generated in control and CCR2 knockout mice then granuloma sizes (upper panel) and compositions (lower panel) were determined over the time course of development. Note selective abrogation of monocyte recruitment in the early stages of granuloma formation. ${ }^{*} P<0 \cdot 01$.

period. In another study, mice treated with a highly specific rabbit antimurine MCP-3 Ab preparation during type 2 (SEA) granuloma formation demonstrated a selective $40-50 \%$ reduction in eosinophil accumulation on day 4 (Figure 9). This observation would be fully consistent with eosinophil recruitment by way of CCR3 and indicates that MCP-3 contributes to a significant component of granuloma eosinophil content. However, redundant recruitment mechanisms are clearly present, since none of the above manipulations were able to completely deplete a selected leucocyte population.

As noted above, because of the putative association of CCR 8 with Th2 cells, this receptor and its ligands have been proposed as potential contributors to type 2 cytokine mediated immune responses. In order to test this hypothesis, 


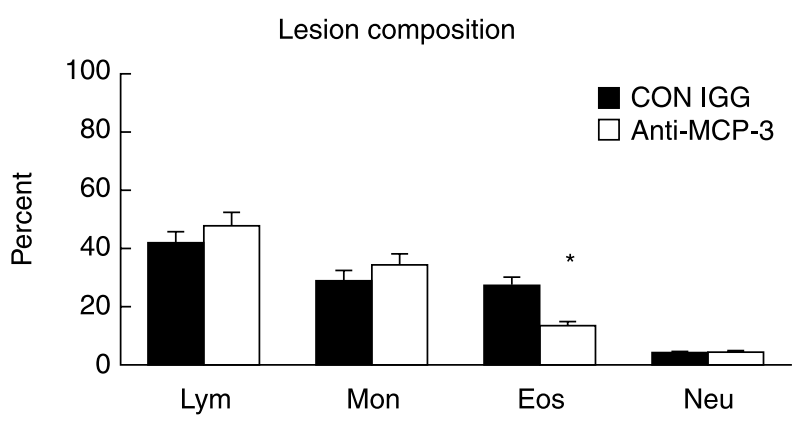

Figure 9 Effect of antibody mediated depletion of monocyte chemotactic protein-3 on schistosomal egg antigen-elicited granuloma composition. Mice were treated with $10 \mathrm{mg}$ of nonimmune or anti-MCP-3 rabbit immunoglobulin G just prior to type 2 (SEA) granuloma induction. On day 4 of development granulomas were isolated, dispersed and compositions determined by direct differential analysis. ${ }^{*} P<0 \cdot 01$.

we recently examined the effect of CCR 8 knockout on granuloma formation (44). That study revealed a defect in eosinophil recruitment and a reduction in type 2 cytokine expression (Figure 10). In contrast, the type 1 (PPD) response cytokines were unaffected. This result was consistent with the reported association of CCR 8 with Th2 cell clones $(27,45,46)$ but our transcript analysis (Figure 6a) indicated that CCR8 and potential CCR8 agonists were likely expressed in both type 1 and type 2 granuloma formation. Why then was the type 2 response selectively affected? In addition, based upon CK receptor studies of $\mathrm{Th} 2$ clones,

Lesion composition
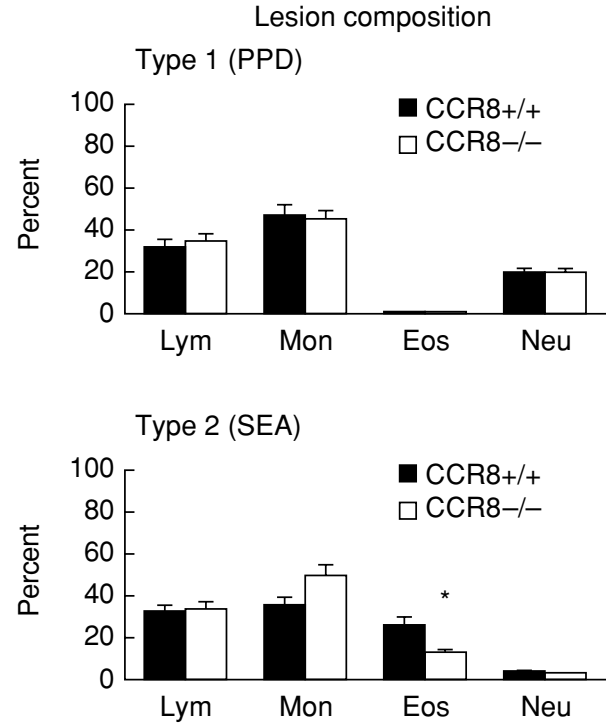

alternative receptors such as CCR3 and CCR4 should have provided compensatory chemotactic responses.

\section{CHEMOKINE RECEPTOR TRANSCRIPT PROFILES OF DRAINING LYMPH NODE CD4 ${ }^{+} \mathrm{T}$ CELLS GENERATED DURING GRANULOMA FORMATION}

Th1 cells generated in vitro reportedly have restricted expression of CXCR 3 and CCR5 whereas Th2 cells express CCR3, CCR4 and CCR8 (47). In order to explore the potential repertoire of $\mathrm{CK}$ responses among $\mathrm{CD}^{+} \mathrm{T}$ cells generated in vivo, we compared $\mathrm{CK}$ receptor transcript levels among populations of $\mathrm{CD}^{+} \mathrm{T}$ cells purified from the draining lymph nodes of mice with schistosomal or mycobacterial Ag-elicited lung granulomas. These are highly polarized populations by intracellular cytokine staining and cytokine secretion assays (data not shown). $\mathrm{CD}^{+} \mathrm{T}$ cells were purified by negative selection columns immediately or after culture in the presence or absence of specific Ag. As shown in Figure 11, freshly isolated type 1 (PPD) $\mathrm{CD}^{+} \mathrm{T}$ cells displayed enhanced levels of CXCR3, CCR4 and CCR6 transcripts compared to naïve $\mathrm{CD}^{+} \mathrm{T}$ cells (dashed lines). The CXCR3 restriction for type 1 (PPD) cells is similar to that reported for in vitro generated Th1 cells but otherwise the pattern differed. Type 2 (SEA) preparations also differed from expected patterns, displaying only enhanced CCR4 mRNA. After Ag-stimulation, type 1 cells showed strong enhancements of CXCR3 and CCR4 but
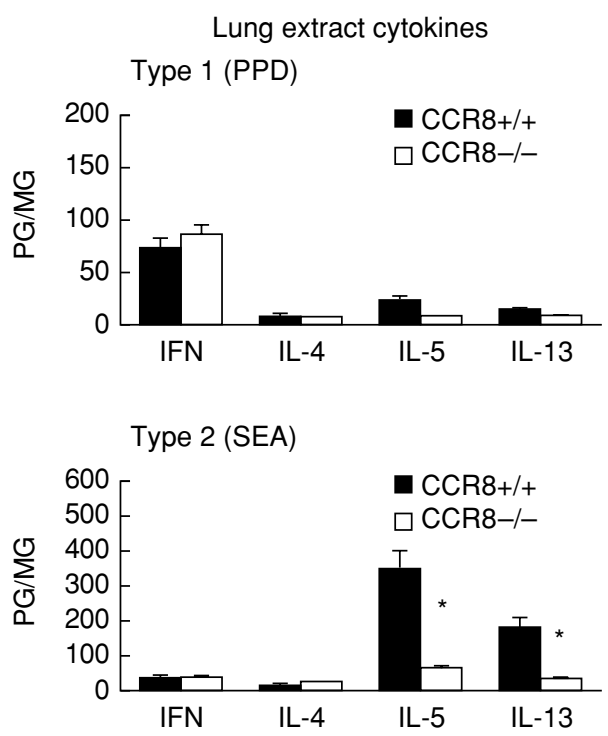

Figure 10 Effect of CC chemokine receptor 8 (CCR8) knockout on mycobacterial and schistosomal egg antigen-elicited granuloma formation. Type 1 (PPD) or type 2 (SEA) bead granulomas were generated in control and CCR8 knockout mice then granuloma compositions (left panels) and lung aqueous extract cytokines (right panels) were determined on day 4 of granuloma formation. Note only the type 2 (SEA) response was affected with reduced eosinophils and type 2 cytokines. $* P<0 \cdot 01$. 
(a)
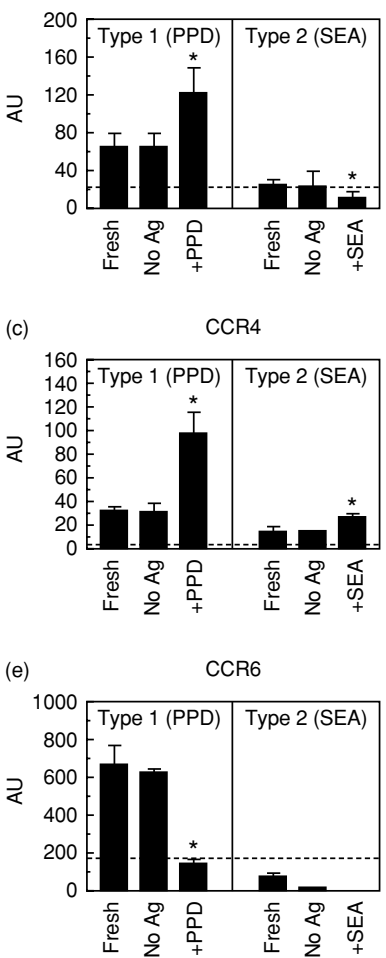

(b)

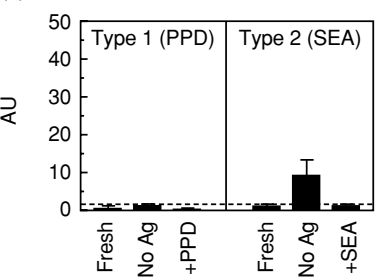

(d)

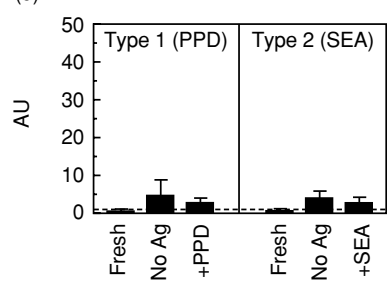

(f)

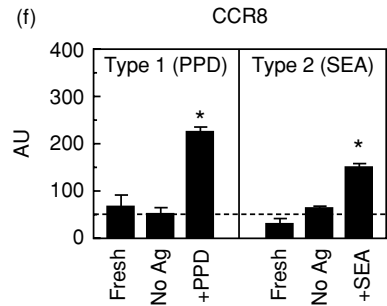

Figure 11 Chemokine receptor transcript expression by freshly isolated and antigen-stimulated draining lymph node $\mathrm{CD}^{+} \mathrm{T}$ cells from mice with type 1 (PPD) and type 2 (SEA) T-cell mediated granuloma formation. Transcript levels were analysed in isolated $\mathrm{CD}^{+} \mathrm{T}$ cells by real time reverse transcriptase-polymerase chain reaction after culture for $48 \mathrm{~h}$ in the absence or presence of $5 \mathrm{mg} / \mathrm{ml}$ PPD or SEA. Bars are mean arbitrary units (AU) \pm SD and derived from two separate experiments. ${ }^{*} P<0.05$ compared to freshly isolated $\mathrm{CD} 4^{+} \mathrm{T}$ cells. Dashed line indicates level of expression by naïve $\mathrm{CD}^{-} \mathrm{T}$ cells.

down-regulation of CCR6 mRNA. In addition, these cells also display a three- to four-fold increase in CCR 8 mRNA. The type 2 schistosomal Ag responsive cells showed two-fold enhancement of CCR4 transcripts but a four- to five-fold increase in CCR8 mRNA. Thus, while CCR8 did not display expression restricted to the type 1 or type 2 response, the type 2 cells were more limited in their repertoire. Thus, type 2 (SEA) responsive $\mathrm{T}$ cells could be more dependent on CCR 8 for functional requirements especially in the postactivation stage.

\section{CONCLUSIONS}

The secondary type 2 schistosomal egg Ag granuloma evokes a characteristic CK profile with expression of CCR2, 3, 4 and 8 ligands but lesser expression of CXCR2, CXCR3, CCR 1 and CCR 5 ligands. Th2 associated cytokines, such as

IL-4 and IL-13, help determine the profile and temporal expression of CKs during schistosomal Ag-elicited granuloma formation. It appears that individual CKs participate at different stages and in different aspects of granuloma formation (e.g. monocyte and eosinophil recruitment and $\mathrm{T}$ cell functional activity) but there is significant redundancy. In vivo generated Th cells may not display precisely the same receptor profiles as those described for artificially generated Th cells. However, functional differences in Th cells may be related to $\mathrm{CK}$ receptor repertoire differences.

\section{ACKNOWLEDGEMENTS}

This work was supported by NIH-NIAID grant AI43460 and in part by the Department of Veteran's Affairs. Schostosome life stages or materials for this work were supplied through NIH-NIAID contract NO1-AI-55270.

\section{REFERENCES}

1 Boros DL, Warren KS. Delayed hypersensitivity-type granuloma formation and dermal reaction induced and elicited by a soluble factor isolated from Schistosoma mansoni eggs. $J$ Exp Med 1970; 132: 488-507.

2 Warren KS. A functional classification of granulomatous inflammation. Ann NY Acad Sci 1976; 278: 7-18.

3 Boros DL. T helper cell populations, cytokine dynamics, and pathology of the schistosome egg granuloma. Microbes Infect 1999; 1: 511-516.

4 Sher A, Coffman RL, Hieny S, Scott P, Cheever AW. Interleukin 5 is required for the blood and tissue eosinophilia but not granuloma formation induced by infection with Schistosoma mansoni. Proc Natl Acad Sci USA 1990; 87: 61-65.

5 Chensue SW, Terebuh PD, Warmington KS et al. Role of IL-4 and IFN-gamma in Schistosoma mansoni egg-induced hypersensitivity granuloma formation. Orchestration, relative contribution, and relationship to macrophage function. $J$ Immunol 1992; 148: 900-906.

6 Vella AT, Hulsebosch MD, Pearce EJ. Schistosoma mansoni eggs induce antigen-responsive CD44-hi $\mathrm{T}$ helper 2 cells and IL-4-secreting CD44-lo cells. Potential for T helper 2 subset differentiation is evident at the precursor level. J Immunol 1992; 149: $1714-1722$.

7 Cheever AW, Williams ME, Wynn TA et al. Anti-IL-4 treatment of Schistosoma mansoni-infected mice inhibits development of $\mathrm{T}$ cells and non-B, non-T cells expressing Th 2 cytokines while decreasing egg-induced hepatic fibrosis. J Immunol 1994; 153: $753-759$.

8 Chensue SW, Warmington KS, Ruth J, Lincoln PM, Kunkel SL. Cross-regulatory role of interferon-gamma (IFN-gamma), IL-4 and IL-10 in schistosome egg granuloma formation: in vivo regulation of $\mathrm{Th}$ activity and inflammation. Clin Exp Immunol 1994; 98: 395-400.

9 Kaplan MH, Whitfield JR, Boros DL, Grusby MJ. Th2 cells are required for the Schistosoma mansoni egg-induced granulomatous response. J Immunol 1998; 160: 1850-1856.

10 Chiaramonte MG, Schopf LR, Neben TY, Cheever AW, Donaldson DD, Wynn TA. IL-13 is a key regulatory cytokine 
for Th2 cell-mediated pulmonary granuloma formation and IgE responses induced by Schistosoma mansoni eggs. J Immunol 1999; 162: 920-930.

11 McKenzie GJ, Fallon PG, Emson CL, Grencis RK, McKenzie AN. Simultaneous disruption of interleukin (IL)-4 and IL-13 defines individual roles in $\mathrm{T}$ helper cell type 2-mediated responses. J Exp Med 1999; 189: 1565-1572.

12 Pearce EJ, La Flamme A, Sabin E, Brunet LR. The initiation and function of Th2 responses during infection with Schistosoma mansoni. Adv Exp Med Biol 1998; 452: 67-73.

13 Chiaramonte MG, Donaldson DD, Cheever AW, Wynn TA. An IL-13 inhibitor blocks the development of hepatic fibrosis during a T-helper type 2-dominated inflammatory response. $J$ Clin Invest 1999; 104: 777-785.

14 Zlotnik A, Yoshie O. Chemokines: a new classification system and their role in immunity. Immunity 2000; 12: 121-127.

15 Rossi D, Zlotnik A. The biology of chemokines and their receptors. Annu Rev Immunol 2000; 18: 217-242.

16 Keane MP, Strieter RM. Chemokine signaling in inflammation. Crit Care Med 2000; 28: N13-N26.

17 Loetscher P, Seitz M, Baggiolini M, Moser B. Interleukin-2 regulates $\mathrm{CC}$ chemokine receptor expression and chemotactic responsiveness in T lymphocytes. J Exp Med 1996; 184: 569577.

18 Bonecchi R, Polentarutti N, Luini W et al. Up-regulation of CCR 1 and CCR 3 and induction of chemotaxis to CC chemokines by IFN-gamma in human neutrophils. J Immunol 1999; 162: 474-479.

19 Chensue SW, Warmington KS, Ruth JH, Lincoln P, Kunkel SL. Cytokine function during mycobacterial and schistosomal antigen-induced pulmonary granuloma formation. Local and regional participation of IFN-gamma, IL-10, and TNF. $J$ Immunol 1995; 154: 5969-5976.

20 Chensue SW, Warmington K, Ruth J, Lincoln P, Kuo MC, Kunkel SL. Cytokine responses during mycobacterial and schistosomal antigen-induced pulmonary granuloma formation. Production of Th1 and Th2 cytokines and relative contribution of tumor necrosis factor. Am J Pathol 1994; 145: 1105-1113.

21 Qiu B, Frait KA, Reich F, Komuniecki E, Chensue SW. Chemokine expression dynamics in mycobacterial (type-1) and schistosomal (type-2) antigen-elicited pulmonary granuloma formation. Am J Pathol 2001; 158: 1503-1515.

22 Park MK, Hoffmann KF, Cheever AW, Amichay D, Wynn TA, Farber JM. Patterns of chemokine expression in models of Schistosoma mansoni inflammation and infection reveal relationships between type 1 and type 2 responses and chemokines in vivo. Infect Immun 2001; 69: 6755-6768.

23 Shang X, Qiu B, Frait KA et al. Chemokine receptor 1 knockout abrogates natural killer cell recruitment and impairs type-1 cytokines in lymphoid tissue during pulmonary granuloma formation. Am J Pathol 2000; 157: 2055-2063.

24 Katschke KJ Jr, Rottman JB, Ruth JH et al. Differential expression of chemokine receptors on peripheral blood, synovial fluid, and synovial tissue monocytes/macrophages in rheumatoid arthritis. Arthritis Rheum 2001; 44: 1022-1032.

25 Sarafi MN, Garcia-Zepeda EA, MacLean JA, Charo IF, Luster AD. Murine monocyte chemoattractant protein (MCP)-5: a novel CC chemokine that is a structural and functional homologue of human MCP-1. J Exp Med 1997; 185: 99-109.

26 D'Ambrosio D, Iellem A, Bonecchi R et al. Selective upregulation of chemokine receptors CCR 4 and CCR 8 upon activation of polarized human type 2 Th cells. J Immunol 1998; 161: 5111-5115.

27 Sallusto F, Kremmer E, Palermo B et al. Switch in chemokine receptor expression upon TCR stimulation reveals novel homing potential for recently activated T cells. Eur J Immunol 1999; 29: 2037-2045.

28 Campbell JD, HayGlass KT. T cell chemokine receptor expression in human Th1- and Th2-associated diseases. Arch Immunol Ther Exp (Warsz) 2000; 48: 451-456.

29 Panina-Bordignon P, Papi A, Mariani M et al. The C-C chemokine receptors CCR4 and CCR8 identify airway $\mathrm{T}$ cells of allergen-challenged atopic asthmatics. J Clin Invest 2001; 107: $1357-1364$

30 Inngjerdingen M, Damaj B, Maghazachi AA. Human NK cells express $\mathrm{CC}$ chemokine receptors 4 and 8 and respond to thymus and activation-regulated chemokine, macrophage-derived chemokine, and I-309. J Immunol 2000; 164: 4048-4054.

31 Haque NS, Fallon JT, Taubman MB, Harpel PC. The chemokine receptor CCR8 mediates human endothelial cell chemotaxis induced by I-309 and Kaposi sarcoma herpesvirus-encoded vMIP-I and by lipoprotein (a)-stimulated endothelial cell conditioned medium. Blood 2001; 97: 39-45.

32 Imai T, Baba M, Nishimura M, Kakizaki M, Takagi S, Yoshie O. The T cell-directed CC chemokine TARC is a highly specific biological ligand for CC chemokine receptor 4. J Biol Chem 1997; 272: 15036-15042.

33 Bernardini G, Hedrick J, Sozzani S et al. Identification of the $\mathrm{CC}$ chemokines TARC and macrophage inflammatory protein1 beta as novel functional ligands for the CCR8 receptor. Eur $J$ Immunol 1998; 28: 582-588.

34 Yoneyama H, Harada A, Imai T et al. Pivotal role of TARC, a CC chemokine, in bacteria-induced fulminant hepatic failure in mice. J Clin Invest 1998; 102: 1933-1941.

35 Caux C, Ait-Yahia S, Chemin K et al. Dendritic cell biology and regulation of dendritic cell trafficking by chemokines. Springer Semin Immunopathol 2000; 22: 345-369.

36 Bowman EP, Campbell JJ, Soler D et al. Developmental switches in chemokine response profiles during B cell differentiation and maturation. $J$ Exp Med 2000; 191: 1303-1318.

37 Fitzhugh DJ, Naik S, Caughman SW, Hwang ST. Cutting edge: C-C chemokine receptor 6 is essential for arrest of a subset of memory $\mathrm{T}$ cells on activated dermal microvascular endothelial cells under physiologic flow conditions in vitro. J Immunol 2000; 165: 6677-6681.

38 Yamashiro S, Wang JM, Yang D, Gong WH, Kamohara H, Yoshimura T. Expression of CCR6 and CD83 by cytokineactivated human neutrophils. Blood 2000; 96: 3958-3963.

39 Cyster JG. Leukocyte migration: scent of the T zone. Curr Biol 2000; 10: R30-R33.

40 Sallusto F, Mackay CR, Lanzavecchia A. The role of chemokine receptors in primary, effector, and memory immune responses. Annu Rev Immunol 2000; 18: 593-620.

41 Chensue SW, Warmington K, Ruth JH, Lukacs N, Kunkel SL. Mycobacterial and schistosomal antigen-elicited granuloma formation in IFN-gamma and IL-4 knockout mice: analysis of local and regional cytokine and chemokine networks. J Immunol 1997; 159: 3565-3573.

42 Ruth JH, Warmington KS, Shang X et al. Interleukin-4 and -13 participation in mycobacterial (type-1) and schistosomal (type2) antigen-elicited pulmonary granuloma formation: multiparameter analysis of cellular recruitment, chemokine expression and cytokine networks. Cytokine 2000; 12: 432-444. 
43 Warmington KS, Boring L, Ruth JH et al. Effect of C-C chemokine receptor 2 (CCR2) knockout on type-2 (schistosomal antigenelicited) pulmonary granuloma formation: analysis of cellular recruitment and cytokine responses. Am J Pathol 1999; 154: 1407-1416.

44 Chensue SW, Lukacs NW, Yang TY, et al. Aberrant in vivo T helper type 2 cell response and impaired eosinophil recruitment in CC chemokine receptor 8 knockout mice. J Exp Med 2001; 193: $573-584$.
45 Zingoni A, Soto H, Hedrick JA et al. The chemokine receptor CCR 8 is preferentially expressed in Th2 but not Th1 cells. $J$ Immunol 1998; 161: 547-551.

46 Napolitano M, Santoni A. Structure and function of the CC chemokine receptor (CCR) 8. Forum 1999; 9: 315-324.

47 Sallusto F, Palermo B, Hoy A, Lanzavecchia A. The role of chemokine receptors in directing traffic of naive, type 1 and type 2 T cells. Curr Top Microbiol Immunol 1999; 246: 123-129. 